\title{
The Effect of Video Education on Skin-to-Skin Contact at the Time of Delivery: A Randomized Controlled Trial
}

\author{
Catherine M. Caponero, DO, MA ${ }^{1,2}$ Dani G. Zoorob, MD, MHA ${ }^{3}$ Victor Heh, PhD 4 \\ Hind N. Moussa, MD 2,5
}

${ }^{1} \mathrm{Ob} /$ Gyn and Women's Health Institute, Cleveland Clinic, Cleveland, Ohio

${ }^{2}$ Department of Obstetrics and Gynecology, Kettering Health, Kettering, Ohio

Address for correspondence Catherine Caponero, DO, MA, Women's Health Institute, Cleveland Clinic, 9500 Euclid Avenue, Cleveland, $\mathrm{OH}$

${ }^{3}$ Department of Obstetrics and Gynecology Academic Offices, 44195 (e-mail: caponeroc27@gmail.com).

University of Toledo, Toledo, Ohio

${ }^{4}$ Office of Academic Affairs, Ohio University College of Osteopathic Medicine, Dublin, Ohio

${ }^{5}$ Division of Maternal-Fetal Medicine, Department of Obstetrics and Gynecology, University of Cincinnati Medical Center, Cincinnati, Ohio

AJP Rep 2022;12:e10-e16.

\begin{abstract}
Keywords

- skin-to-skin contact

- breastfeeding

- video education

- delivery

- prenatal care

Objective The objective of this study was to measure the impact of video education at the time of admission for delivery on intent and participation in skin-to-skin contact (SSC) immediately after birth.

Methods This study was a randomized controlled trial of educational intervention in women $(N=240)$ of 18 years or older admitted in anticipation of normal spontaneous term delivery. Alternate patients were randomized into video $(N=120)$ and no video $(N=120)$ groups. Both groups received a survey about SSC. The video group watched an educational DVD and completed a postsurvey about SSC.

Results During the preintervention survey, $89.2 \%$ of those in the video group compared with $83.3 \%$ of those in the no video group indicated that they planned to use SSC $(p=0.396)$. After the video, 98.3\% planned to do SSC after delivery $(p<0.001)$. However, only $59.8 \%$ started SSC within 5 minutes of delivery in the video group and only $49.4 \%$ started SSC within 5 minutes of delivery in the no video group $(p=0.17)$. Conclusion Video education alters the intention and trends toward participation in SSC within 5 minutes of delivery. Despite the plans for SSC, however, there was no significant difference in rates between the two groups. These findings support that obstacles, other than prenatal education, may affect early SSC.
\end{abstract}

Despite strong support of breastfeeding by organizations such as the American Academy of Pediatrics, the American Congress of Obstetricians and Gynecologists, and the American Academy of Family Physicians, the rate of breastfeeding initiation at the time of delivery and continuation at 6 months postpartum is well below the Center of Disease Prevention's Healthy People 2010 goals. ${ }^{1}$ The United Nations Children's Fund (UNICEF) and the World Health received

July 28,2020

accepted after revision

October 8, 2021
DOI https://doi.org/ $10.1055 / \mathrm{s}-0041-1741540$ ISSN 2157-6998.

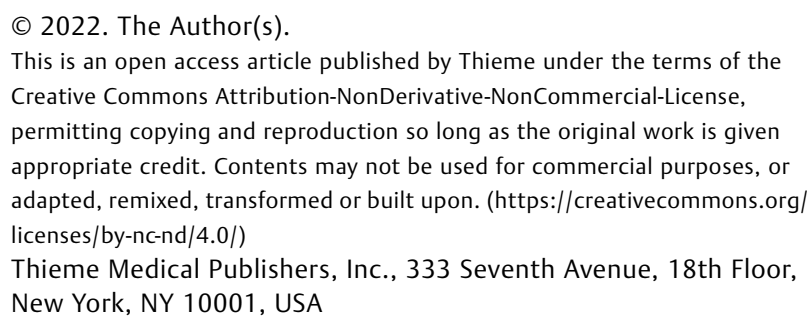




\section{Key Points}

- Significant obstacles impact skin-to-skin rate.

- Video education alters skin-to-skin intent.

- Video education can improve skin-to-skin rate.

- Education can happen at the time of delivery.

- Video education can impact mothers and infants.

Organization (WHO) have estimated that if all infants were breastfed for a minimum of the first 6 months of their lives, the rate of morbidity and malnutrition would significantly decrease across the world. ${ }^{2}$ As a result, WHO and UNICEF launched the Baby-Friendly Hospital Initiative (BFHI) in 1991 to encourage proper infant feeding practices starting at birth. The initiative includes 10 steps which hospitals must meet to obtain and maintain certification. Step 4 of the BFHI aims to "help mothers initiate breastfeeding within 1 hour of birth," and skin-to-skin contact (SSC) between mother and infant within 5 minutes is encouraged and set as the standard criteria in support of this goal. ${ }^{3}$

Skin-to-skin, otherwise known as SSC, on a mother's chest immediately following birth provides thermoregulation, physiological stability, appropriate stimulation for the infant, and encourages bonding and breastfeeding. ${ }^{4}$ Previous work demonstrates that early SSC is positively associated with breastfeeding from discharge through 3 months postpartum. ${ }^{5}$ However, there are significant obstacles to SSC and breastfeeding including insufficient prenatal education, disruptive obstetrical practices, and a lack of family and societal support. ${ }^{6}$ These barriers are more prevalent among vulnerable groups such as black, low income, or poorly educated populations. $^{2}$ Delivery room practices and postpartum hospital routines may also hinder initiation of SSC if not part of the standard care protocols and thus significantly disrupt early maternal-infant interactions. ${ }^{7}$ While initiatives such as BFHI may combat some of these obstacles, guidelines for primary care-based interventions originating in a clinician's office currently do not exist. ${ }^{8}$

In this study, we sought to evaluate the impact of prenatal video education on both pregnant women's intention as well as the actual rate of practicing SSC after birth. We hypothesized that by providing SSC education in an easily comprehensible and patient-friendly video format shortly before delivery would encourage participation in SSC immediately upon delivery. This timing would allow the topic to be remembered clearly and would allow patients to engage in an active dialogue with the staff who will be preforming the delivery. ${ }^{9}$ The goal of educating mothers was not only to increase their knowledge and skills but also to influence their attitudes toward accepting early SCC. ${ }^{10}$ By providing a video which models SSC to all nonemergent anticipated vaginal deliveries upon admission to the hospital, our hope was to encourage patients to actively participate in SSC at the time of delivery and become their own advocates for such a practice.

\section{Materials and Methods}

\section{Patient Selection}

Study participants were recruited at the time of admission to labor and delivery by an obstetrics and gynecology resident at Southview Medical Center. Patients met inclusion criteria for this study if they were admitted with anticipation of a normal spontaneous vaginal delivery within 1 week of the time of admission, were more than 37 weeks of gestational age, fluent in spoken English, and older than 18 years. Patients who did not meet the aforementioned criteria or who were unstable medically at the time of delivery were excluded from the study.

\section{Study Design}

Following recruitment, patients were immediately randomized into no video (Group A-control) and video groups (Group B-experimental). Patients assigned to Group B watched a video titled "Jumping into Kangaroo Care" developed by the Ohio Department of Health. The video is 8 minutes and 52 seconds in length. It discusses the benefits and logistics of kangaroo care, a commonly used methodology for education and awareness related to SSC, from both the professional and new mother's perspectives.

Randomization occurred in the order that the patient was enrolled in the study. The first patient enrolled was in Group A and asked a presurvey regarding:

- their intention to practice SSC at the time of delivery

- if they participated in SSC in a previous pregnancy

- if they had any formal training about SSC

- and if they did have formal training, was it either

- provided at a prenatal appointment

- or a formal class led by either a nurse or a lactation consultant

The second patient enrolled was in Group B and took the same presurvey, watched the "Jumping into Kangaroo Care" video, and then took the postsurvey which asked if they intended to practice SSC at the time of delivery. The third patient enrolled would take only the presurvey and be in Group A, etc. The complete survey is shown in -Appendix A.

This project was approved by the Institutional Review Board at Kettering Health Network prior to registration with ClinicalTrials.gov (study number NCT03200925).

\section{Outcome Measures}

Our primary outcome of interest was the intent to participate in SSC following delivery. Our secondary outcome was 
participation in SSC within 5 minutes of delivery. To identify significant differences in time until start of SSC, this variable was dichotomized to "within 5 minutes" and "after 5 minutes."

\section{Data Collection}

For each participant, we collected data on patient age, gestational age at enrollment, pregnancy comorbidities, race, type of insurance, and the number of times the patient has been pregnant. We also collected the neonate's 5-minute Apgar score, delivery date/time, SSC initiation time, SSC end time, delivery to SSC duration (in minutes), and SSC duration (in minutes). Finally, we determined if SSC was not initiated because of new-onset increased level of fetal or maternal acuity of care, or if the mother declined/elected not to proceed with SCC. These data were obtained through routine nursing documentation as apart of standard workflow. If a participant delivered with a 5-minute Apgar less than 7 or required a cesarean delivery, the patient was not included in data collection regarding SSC at the time of delivery. Since the primary outcome was intent to perform SCC, analysis was performed for this group only on this basis and not on actual implementation of SCC.

\section{Statistical Analysis}

\section{Sample Size Calculation}

In the quarter prior to this study, Southview Medical Center had established that the institutional baseline of SSC was 50\% for eligible vaginal deliveries (i.e., more than 37 weeks' gestation with a 5-minute Apgar of 7). The BFHI had set a goal for the hospital to achieve $82 \%$ rate of SSC.

We employed planned interim analysis based on a recruitment goal of 240 patients and adopted "two looks" at the data to determine statistical significance. This was completed by our statistician who was blinded to the study participants. The first look occurred after recruiting 120 women (60 per group) and the second look after reaching the goal of 240 women (120 per group). We used cutoff points of $z=3.5$ for first look and $z=2.0$ for second look. ${ }^{11}$ Hypothetically, $50 \%$ of Group A and $80 \%$ of Group B intended to participate in SSC (30\% difference in proportion) during the first interim analysis, 50\% interim sample size (120 patients) would result in an absolute $z$-statistics of 4.89 , and data collection would have ended. However, given our smaller difference in proportions on first look, data collection was continued until 240 patients were recruited to reach statistical significance.

Descriptive characteristics were presented as mean ( \pm standard deviation) for continuous data and sample size (percentage) for categorical data. Continuous variables were compared using $z$-test and categorical variables were compared using chi-square test. Dichotomous outcomes were further analyzed using logistic regression with adjustment for clinically and statistically significant variables. All statistical tests were two sided, power of $80 \%$, and $p$-values less than 0.05 were considered statistically significant. SPSS (IBM Corp.) version 22 was used for data analysis.

\section{Results}

\section{Intention to Participate in SSC before and after Video Education}

There were no significant differences between the two randomized groups of patients in terms of baseline characteristics such as demographics, comorbidities, and obstetric measures (-Table 1). Most respondents in both groups indicated that they planned to use SSC after delivery. The preintervention survey showed that there was no difference in intent to use SSC between the two groups with $89.2 \%$ ( $n=107 / 120)$ of the video group indicating intent to use SSC compared with $83.3 \%(n=100 / 120)$ of the no video group $(p=0.396)$ ( - Fig. 1). However, after watching the video, the percentage of patients intending to use SSC after delivery increased to $98.3 \%(118 / 120 ; p<0.001)$ (-Table 2 ).

\section{Effect of Video Intervention on Time from Delivery to Start of SSC}

Of the 240 patients included in the study, 63 patients proceeded to cesarean section or did not complete SSC due to increased fetal/maternal acuity of care, or maternal refusal. As such, 177 (73.8\%) were included in the analysis for time to SSC per se (-Fig. 1). The video group trended toward initiating SSC within 5 minutes of delivery at $59.8 \%$ ( $n=55 / 92$ ) compared with the rate in the no video group of $49.4 \%(n=42 / 85)(p=0.17)$.

\section{Effect of Prior Formal Education by Physician/Nurse/ Lactation Consultant on Time to SSC}

Of the 177 patients, 58(32.8\%) reported formal education before they were included in the study, and 119 (67.2\%) did not report any formal education ( - Table 2 ). The proportion of those who reported formal education beforehand started SSC within 5 minutes was $62.1 \%(n=36 / 58)$, while in those who did not report formal education, it was $51.3 \%(n=61 / 119)(p=0.18)$.

A subanalysis was performed for only those who did not have any formal education before joining the study $(n=119)$. This demonstrated that a greater proportion of patients in the video group who initiated SSC within 5 minutes of delivery compared with those in the no video group (59.7\% [ $n=37 / 62]$ vs. $42.1 \%[n=24 / 57], p=0.04)$.

\section{Comments}

\section{Principal Findings/Results}

In this study, we found that the video education intervention increased intent to perform SSC in pregnant patients receiving that education upon their delivery hospitalization. Furthermore, SCC completion rates were increased after that video education in women who did not perceive having prior formal education about SCC during their pregnancy. To date, this is the only study that examines the impact of education at that time on intent and implementation of SCC.

\section{Clinical Implications}

If we consider efforts made to improve breastfeeding rates, we see that patient education programs have the single 
Table 1 Patient characteristics and obstetric measures for those who did and did not receive video training on SSC

\begin{tabular}{|c|c|c|c|}
\hline Patient Characteristics & Video group, $N=120$ & No video group, $N=120$ & $p$-Value \\
\hline \multicolumn{4}{|l|}{ Demographic } \\
\hline Age, mean (SD) & $27.0(5.1)$ & $27.1(5.5)$ & 0.9 \\
\hline Primiparous, $n(\%)$ & $55(45.8)$ & $53(44.2)$ & 0.8 \\
\hline Caucasian, $n(\%)$ & $98(81.7)$ & $90(75.0)$ & 0.21 \\
\hline Private insurance, $n(\%)$ & $58(48.3)$ & $55(45.8)$ & 0.92 \\
\hline Medicaid, $n(\%)$ & $58(48.3)$ & $61(50.8)$ & \\
\hline \multicolumn{4}{|l|}{ Comorbidities } \\
\hline Chronic hypertension, $n(\%)$ & $3(2.5)$ & $5(4.2)$ & 0.47 \\
\hline Gestational hypertension, $n(\%)$ & $10(8.3)$ & $6(5.0)$ & 0.3 \\
\hline Preeclampsia, $n(\%)$ & $2(1.7)$ & $3(2.5)$ & 0.65 \\
\hline Gestational diabetes mellitus, $n(\%)$ & $9(7.5)$ & $4(3.4)$ & 0.16 \\
\hline Asthma, $n(\%)$ & $10(8.3)$ & $10(8.3)$ & 1 \\
\hline Neurologic disease, $n$ (\%) & $1(0.8)$ & $4(3.8)$ & 0.18 \\
\hline Hematologic disease, $n(\%)$ & $9(7.5)$ & $13(10.8)$ & 0.37 \\
\hline Other comorbidities, $n(\%)$ & $42(35.0)$ & $40(33.3)$ & 0.79 \\
\hline \multicolumn{4}{|l|}{ Obstetric measures } \\
\hline Spontaneous vaginal delivery, $n(\%)$ & $89(74.2)$ & $84(70.0)$ & 0.77 \\
\hline Vacuum delivery, $n(\%)$ & $9(7.5)$ & $11(9.2)$ & 0.82 \\
\hline Low transverse cesarean delivery, $n$ (\%) & $22(18.3)$ & $25(20.8)$ & 0.75 \\
\hline Gestational age in wk, mean (SD) & 39 & 39 & 1.00 \\
\hline 5-min Apgar, median & $9(8,10)$ & $9(7,9)$ & 0.16 \\
\hline
\end{tabular}

Abbreviations: SD, standard deviation; SSC, skin-to-skin contact, median (minimum, maximum).

greatest effect of any single intervention on both initiation and short-term duration for breastfeeding. ${ }^{8}$ For example, women who attend breastfeeding classes with lactation consultants are more likely to continue breastfeeding at 6 months when compared with controls. ${ }^{1}$

Although $91 \%$ of the cohort "planned" to initiate SSC immediately after delivery, only $60 \%$ of eligible individuals in the video group started SSC within 5 minutes of delivery and only $50 \%$ started SSC in the no video group followed suit. Women without prior formal education about SCC were more likely to initiate SSC within 5 minutes of delivery after the video education intervention during the delivery hospitalization. However, the "lower than planned" rates likely occurred due to barriers to SSC independent of patient education, including but not limited to staff education and the clinical culture.

Patient education can easily influence behaviors without requiring substantial effort on behalf of providers, and different options must be considered when attempting to improve SSC rates. ${ }^{9}$ Currently, common office practices include provision of written materials and discharge packets. However, neither practice effectively increases rates of breastfeeding, and discharge packets have even reported to actually reduce the rates of breastfeeding. ${ }^{8}$ As such, written documents would not likely improve SSC rates. Ideally, antepartum appointments should include education on the benefits of both breastfeeding and SSC. ${ }^{8}$ However, in a busy clinic setting with a vulnerable patient population, the ability to provide extra educational classes is not always an option, and this study demonstrates the alternative option of video education at the time of delivery. The working premise is that by watching the video shortly before delivery, patients will have the topic of SSC fresh in their minds when it comes time to decide on how to interact with the neonate. Video intervention may also be an effective approach for other perinatal interventions, including breastfeeding, diet/exercise, immunizations, and smoking cessation.

As demonstrated in this study, video education has the potential to not only inform but also effect change. Video has the capability to present a lot of complex information in a rapid, simple, and clear manner. ${ }^{12,13}$ One study found that patients who viewed videotapes that discussed treatment options had a greater understanding of the risks and benefits of those choices and were more apt to be active participants in decision-making. ${ }^{12}$ Audio-visual material can also be useful for those who have limited literacy skills. Moreover, the information provided to patients in video format has the advantage of being consistent when repeated, which allows practitioners to provide the same information to all patients. ${ }^{14}$ This type of intervention is ideal for the perinatal setting given the need to provide accurate information in a concise, expeditious, and standardized manner that applies to the majority of pregnant patients. 
e14 The Effect of Video Education on Skin-to-Skin Contact Caponero et al.

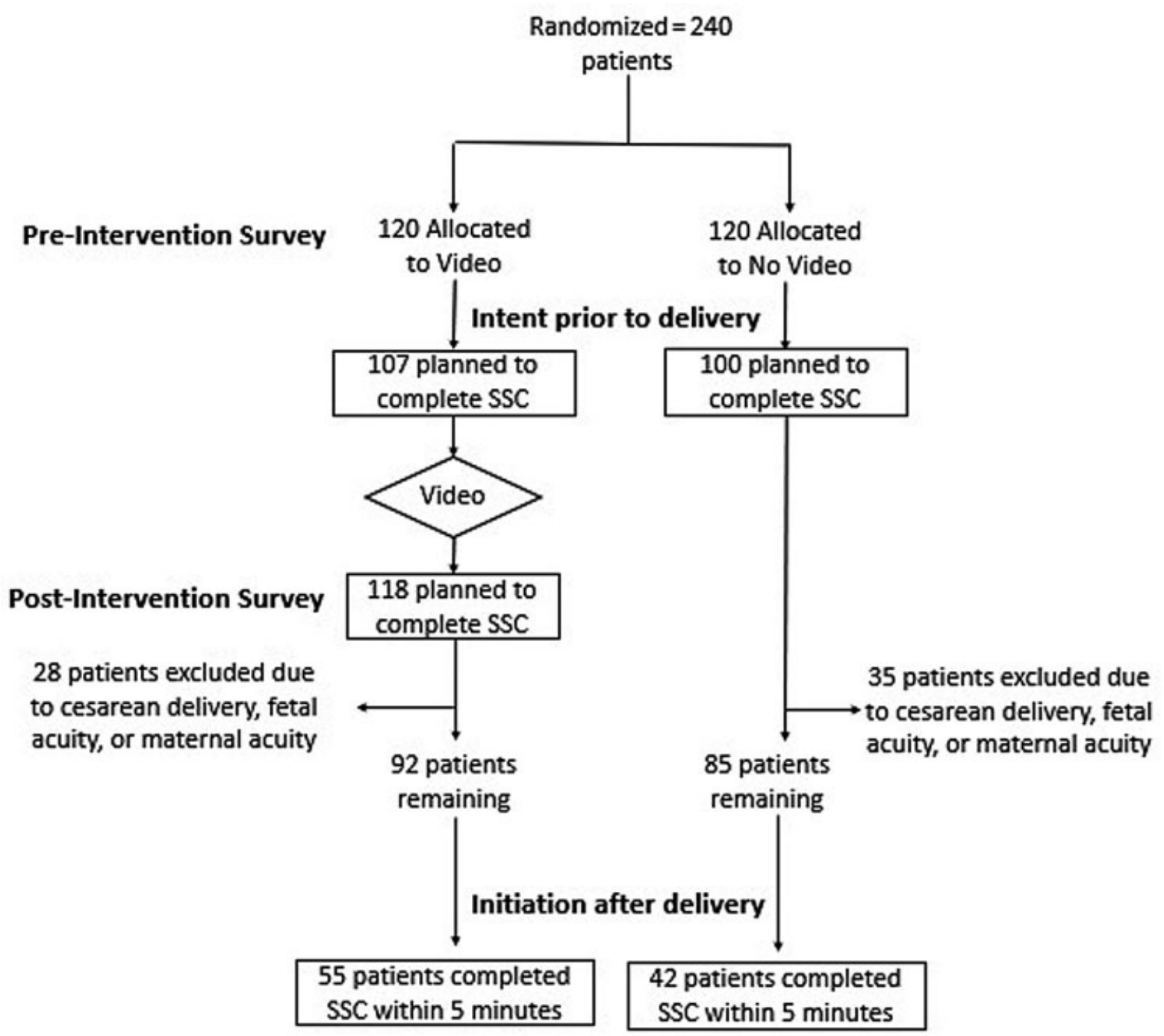

SSC $=$ Skin-To-Skin Contact

Fig. 1 CONSORT diagram. SSC, skin-to-skin contact.

Table 2 Outcome measures for those who did and did not receive video training on SSC

\begin{tabular}{|l|l|l|l|}
\hline Outcome measures & $\begin{array}{l}\text { Video group, } \\
N=120\end{array}$ & $\begin{array}{l}\text { No video group, } \\
N=120\end{array}$ & $p$-Value \\
\hline SSC intention pre, yes, $n(\%)$ & $107(89.2)$ & $100(83.3)$ & 0.19 \\
\hline SSC intention post, yes, $n(\%)$ & $118(98.3)$ & - & - \\
\hline Prior formal education about SSC & $38(31.7)$ & $40(33.3)$ & 0.78 \\
\hline Patients studied for initiation of SSC & $n=92$ & $n=85$ & 0.17 \\
\hline Delivery to SSC in min, median & $3(0,26)$ & $6(0,39)$ & 0.19 \\
\hline SSC duration in min, median & $91(13,210)$ & $88(17,161)$ & 0.88 \\
\hline
\end{tabular}

Abbreviations: SD, standard deviation; SSC, skin-to-skin contact, median (minimum, maximum).

\section{Research Implications}

With SSC implemented at a less than planned rate, future studies should involve other barriers including staff and family education. Timing of education in other settings as well, such as throughout prenatal care or in group prenatal care models, could also be considered in future studies.

\section{Limitations and Strengths}

This study has several limitations and strengths that are worth noting. One limitation was that we could not control patients' previous exposure to SSC training, nor the influence of delivery room personnel including physicians, nursing staff, respiratory therapists, residents, students, and patient's families on initiation of SSC. This, however, is 
consistent with standard clinical practice and thus our population can reflect the normal population with similar characteristics. Another limitation was that the time to SSC analysis was limited to healthy infants with Apgar greater than 7, vaginal deliveries, and infants being born after 37 weeks, and as such it is notable that many patients were not able to have their intentions fulfilled. We also utilized a pragmatic design for randomization; however, the data show that the groups were statistically similar. Importantly, this trial has several strengths. The first is that it is a randomized controlled trial with statistically similar groups. The second is that after an extensive PubMed search, no other studies were found that examined the impact and timing of patient education on SSC.

\section{Conclusion}

Through this randomized controlled study, we can conclude that video education at the time of admission improves a patient's intent to participate in SSC. These findings support that additional barriers, other than prenatal education, may affect a patient's completion of SSC. In women without perceived prior education about SSC, the video intervention was successful in improving SSC rates. That said, this study provides an easily implementable tool that can motivate new mothers to engage in evidence-driven practice with the potential to improve both mother and infant well-being.

Oral Presentations

ACOG Districts VEVII Annual Meeting on Sunday, September 23, 2018, in Kansas City, MO; The ACOOG 86th Annual Conference on Thursday, March 28, 2019, in New Orleans, LA; and The First Annual Network Research Symposium on Friday, May 3, 2019, in Kettering, $\mathrm{OH}$.

\section{Poster Presentations}

The ACOOG 86th Annual Conference on Monday, March 25, 2019, in New Orleans, LA; DAGMEC 20th Annual Virginia C. Wood Resident Research Forum on Thursday, April 25, 2019, in Dayton, OH; The First Annual Network Research Symposium on Friday, May 3, 2019, in Kettering, OH; and The 2019 ACOG Annual Clinical and Scientific Meeting on Sunday, May 5, 2019, in Nashville, TN.

\section{Poster Publication}

2019 MEFACOOG Annual Report.

Clinical Trial Registration

https://clinicaltrials.gov/ct2/show/NCT03200925

\section{Authors' Contribution}

C.M.C., is the project lead and manuscript initial write-up; D.G.Z. contributed in manuscript editing; V.H. contributed in statistics; and H.N.M. is the mentor and faculty advisor, manuscript write-up.

Conflict of Interest

None declared.

\section{References}

1 Rosen IM, Krueger MV, Carney LM, Graham JA. Prenatal breastfeeding education and breastfeeding outcomes. MCN Am J Matern Child Nurs 2008;33(05):315-319

2 Burgio MA, Laganà AS, Sicilia $\mathrm{A}$, et al. Breastfeeding education: where are we going? A systematic review article. Iran J Public Health 2016;45(08):970-977

3 Baby-Friendly USA. "Guidelines and Evaluation Criteria for Facilities Seeking Baby-Friendly Designation.". Albany, NY: Baby-Friendly USA; 2016

4 Charpak N, Ruiz JG. Latin American Clinical Epidemiology Network Series - Paper 9: the kangaroo mother care method: from scientific evidence generated in Colombia to worldwide practice.J Clin Epidemiol 2017;86:125-128

5 Vila-candel R, Duke K, Soriano-vidal FJ, Castro-sánchez E. Affect of early skin-to-skin mother-infant contact in the maintenance of exclusive breastfeeding: experience in a health department in Spain. J Hum Lact 2018;34(02):304-312

6 Chung M, Raman G, Trikalinos T, Lau J, Ip S. Interventions in primary care to promote breastfeeding: an evidence review for the U.S. Preventive Services Task Force. Ann Intern Med 2008;149(08):565-582

7 Moore ER, Anderson GC, Bergman N. Early skin-to-skin contact for mothers and their healthy newborn infants. Cochrane Database Syst Rev 2007;(03):CD003519

8 Guise JM, Palda V, Westhoff C, Chan BK, Helfand M, Lieu TAU.S. Preventive Services Task Force. The effectiveness of primary carebased interventions to promote breastfeeding: systematic evidence review and meta-analysis for the US Preventive Services Task Force. Ann Fam Med 2003;1(02):70-78

9 Martínez-Galiano JM, Delgado-Rodríguez M. Influence of an education program of pregnant women on delivery. J Matern Fetal Neonatal Med 2014;27(07):719-723

10 Shealy KR, Li R, Benton-Davis S, Grummer-Strawn LM. The CDC Guide to Breastfeeding Interventions. Atlanta: U.S. Department of Health and Human Services, Centers for Disease Control and Prevention; 2005

11 O'Brien PC, Fleming TR. A multiple testing procedure for clinical trials. Biometrics 1979;35(03):549-556

12 Krouse HJ. Video modelling to educate patients. J Adv Nurs 2001; 33(06):748-757

13 Hurtubise L, Martin B, Gilliland A, Mahan J. To play or not to play: leveraging video in medical education. J Grad Med Educ 2013;5 (01):13-18

14 Abu Abed M, Himmel W, Vormfelde S, Koschack J. Video-assisted patient education to modify behavior: a systematic review. Patient Educ Couns 2014;97(01):16-22 
e16 The Effect of Video Education on Skin-to-Skin Contact Caponero et al.

Appendix A Survey questions for: the effect of video education on skin-to-skin contact at the time of delivery

\begin{tabular}{|c|c|}
\hline \multirow[t]{4}{*}{$\begin{array}{l}\text { Survey questions asked at the time } \\
\text { of admission by both Groups } A+B\end{array}$} & $\begin{array}{l}\text { Do you plan on participating in skin-to-skin contact immediately after delivery? } \\
\text { a. Yes } \\
\text { b. No } \\
\text { c. I do not know }\end{array}$ \\
\hline & $\begin{array}{l}\text { Have you participated in skin-to-skin contact in a previous delivery? } \\
\text { a. Yes } \\
\text { b. No } \\
\text { c. I do not know } \\
\text { d. I have never had an infant before }\end{array}$ \\
\hline & $\begin{array}{l}\text { Have you had formal education about skin-to-skin contact? } \\
\text { a. Yes } \\
\text { b. No }\end{array}$ \\
\hline & $\begin{array}{l}\text { If completed formal education, was it either } \\
\text { a. Provided at a prenatal appointment by a physician or nurse } \\
\text { b. A formal class led by either a nurse or a lactation consultant }\end{array}$ \\
\hline $\begin{array}{l}\text { Survey question asked to Group B } \\
\text { after video education }\end{array}$ & $\begin{array}{l}\text { Do you plan on participating in skin-to-skin contact immediately after delivery? } \\
\text { a. Yes } \\
\text { b. No } \\
\text { c. I do not know }\end{array}$ \\
\hline
\end{tabular}

